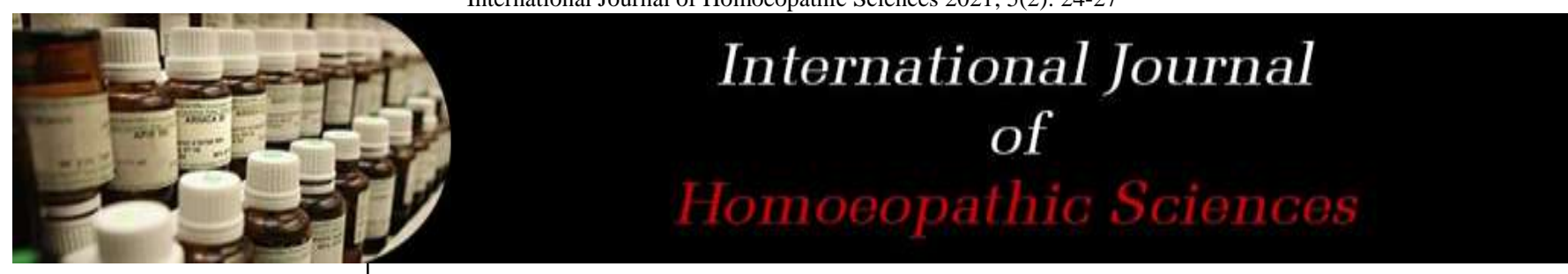

E-ISSN: 2616-4493 P-ISSN: 2616-4485 www.homoeopathicjournal.com IJHS 2021; 5(2): 24-27

Received: 19-05-202

Accepted: 23-06-2021

Dr. Julka Kumari Tripathi 21st Batch PGT, National Institute of Homoeopathy, Kolkata, West Bengal, India

Corresponding Author: Dr. Julka Kumari Tripathi 21st Batch PGT, National Institute of Homoeopathy, Kolkata, West Bengal, India

\section{History of hygiene and homoeopathy}

\section{Dr. Julka Kumari Tripathi}

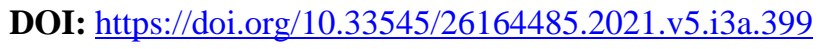

\section{Abstract}

There is obvious and direct relationship between improvement in general levels of cleanliness in society and improved health. It is interesting to see how development of homoeopathy and hygiene both are positively affected each other. The goal of medicine is not only to treat sickness but also prevention, promotion and improvement of the quality of life and health of individual or group or community so maintenance of general hygiene and sanitation, regular bath and care of skin Hygiene of food \& water, to avoid various infections, and Hahnemann's writing clearly demonstrate that his view on importance of hygiene, diet, public health, healthy mode of living, treatment and prevention of various diseases, among which Cholera occupies special attention as here Homoeopathy has showed its triumph all over the world.

Keywords: Hygiene, homoeopathy, hygiene hypothesis

\section{Introduction}

Hygiene refers to conditions and practices that help to maintain health and prevent the spread of diseases ${ }^{[1]}$

The works of Master Hahnemann was much ahead of his time of physician regarding the hygiene and concept of disease prevention. Prevention of Scarlet fever, Cholera, Purpura miliaris etc are striking examples that he had shown to the world.

In the 1796 Samuel Hahnemann published his first treatise about a new principle of medication i.e., Homoeopathy. Even before the book was published in 1792 at Frankfort, "Friend of health", a book written by Hahnemann before the discovery of homeopathy, is considered as a book on preventive medicine in which Hahnemann "recommended the use of fresh air, bed rent proper diet, sunshine, public hygiene and numerous other beneficial measures at a time when many other physicians considered them of no value.

The most convincing evidence of the benefits of handwashing for the general public is for prevention of infectious agents found transiently on hands or spread by the fecal-oral route or from the respiratory tract.

History ${ }^{[2,3,4,5]}$.

The earliest written account of elaborate codes of hygiene can be found in several Hindu texts, such as the Manusmriti and the Vishnu Purana. Bathing is one of the five Nitya karmas (daily duties) in Hinduism, and not performing it leads to sin, according to some scriptures. Ayurveda is a system of medicine developed in ancient times that is still practiced in India, mostly combined with conventional Western medicine. Contemporary Ayurveda stresses a sattvic diet and good digestion and excretion. Hygiene measures include oil pulling, and tongue scraping.

Islamic hygienical jurisprudence, which dates back to the $7^{\text {th }}$ century, has a number of elaborate rules. Taharah (ritual purity) involves performing wudu (ablution) for the five daily salah (prayers), as well as regularly performing ghusl (bathing), which led to bathhouses being built across the Islamic world. In the $9^{\text {th }}$ century, Ziryab invented a type of deodorant to get rid of bad odors.

The teachings of Lord Buddha as well as the Bible and Koran covered various aspects of personal hygiene and other public health practices.

During the ancient Egyptian period developments such as toilets and bathing were introduced, but this was on a private level.

The Greeks encouraged healthy living and pursued regimens of exercise and hygiene such as those prescribed by Hippocrates in his Regimen and Regimen in Acute Diseases (4th century 
BC). Hippocrates (460 BC to $377 \mathrm{BC}$ ) exhorted that to stay healthy, one needed order and balance in all things and, above all, to stay away from the 'Airs, Waters and Places' that contained the dangerous miasmas that were responsible for disease

In the late $18^{\text {th }}$ century Hahnemann, the founder of homoeopathy took a prominent part to enlighten the Government of the day, by his numerous writings e.g.,

- Protection against infection in epidemic diseases.

- Plans for eradicating malignant Fever (in a letter to the Minister of Police).

- Suggestions for the prevention of Epidemic in general specially in Towns.

All these articles appeared in the "Friend of Health" (Hufeland's leading medical Journal of his time) between 1792-1795.

Hahnemann says, the physician is not only a healer but also a preserver of health. The mission of the physician is not just curing the sick individual, but even preventing the people from becoming sick. By guiding on sanitation and hygiene a physician can fulfill his duties completely.

In mid- $19^{\text {th }}$ century England, outbreaks of cholera led to an epidemic in 1854. A physician called John Snow observed that cholera seemed to spread via sewage-contaminated water. This was mostly noticed around a water pump in Broad Street, London. John removed the pump handle and the spread was instantly contained. Until this time, only the elite in Western cities typically possessed indoor facilities for relieving bodily functions. The poorer majority used communal facilities built above cesspools in backyards and courtyards. This changed after Dr. John Snow discovered that cholera was transmitted by the fecal contamination of water.

Greene ${ }^{[6]}$ used historical and cross-cultural evidence and causal inference to associate personal hygiene with better health. However, the role of personal cleanliness ${ }^{[7]}$ in the control of infectious diseases over the past century is difficult to measure, since other factors have changed at the same time (e.g., improved public services, waste disposal, water supply, commercial food handling, and nutrition) ${ }^{[8]}$

Chadwick has generally been considered to be the person who defined prevention of disease in "Sanitary" terms.

Louis Pasteur's experiments proved the germ theory of disease and Joseph Lister and others put them into practice in sanitation, hygienic practices came to be regarded as synonymous with health, as they are in modern times

Lysol (1920) was sold as a genital disinfectant and birth control method. Lysol ads proclaimed a host of benefits for every gynaecological need, and was the leading form of birth control from 1930 to 1960.

Definition ${ }^{[9]}$ : The word hygiene is derived from the name Hygeia, who was the Greek goddess of healing. In modern usage hygiene usually refers to "Cleanliness and especially to any practice which leads to the absence or reduction of harmful infectious agents."

Classification of Hygiene ${ }^{[10]}$.

Hygiene can be classified in two aspects

- Personal

- Environmental
Personal Hygiene: Its aim is to promote standards of personal cleanliness within the setting of the condition where people live. Personal Hygiene includes bathing, clothing, washing hands \& toilets; care of nails, feet \& teeth; spitting, coughing, sneezing, personal appearance \& inculcation of clean habits in the young. Training in this field should begin at a very early age $\&$ must be carried through school age.

Environmental Hygiene: Improvement of environmental health is a major concern of many governments \& related agencies throughout the world. This has two aspects

- Domestic Hygiene: Comprises home, use of soap, need for fresh air, light \& ventilation; hygienic storage of foods, hygienic disposal of wastes, need to avoid pests, rats, mice $\&$ insects.

- Community Hygiene: Emphasis is given on basic sanitary services which consist of water supply, disposal of human excreta, other solid \& liquid wastes, vector control, food sanitation \& housing which are fundamental to health.

\section{Hand hygiene ${ }^{[11]}$}

Master Hahnemann in friends of health advised that after every visit the medical officers should wash their hands and faces in vinegar and water also If he requires to feel the pulse, he must do this with averted head, and immediately afterwards wash his hand in a basin containing water and vinegar. In 1847 Hungarian physician Dr Ignaz Semmelweis, working at the Vienna Maternity Hospital, made the link between the prevalence of puerperal (childbed) fever and dirty hands. He ordered doctors, nurses and midwives to wash their hands in chlorinated water before contact with each patient. Despite a dramatic decrease in the death rate, he was ridiculed for many years. Hand hygiene is the most effective measure for interrupting the transmission of microorganisms which cause infection both in the community and in the healthcare setting. Using hand hygiene as a sole measure to reduce infection is unlikely to be successful when other factors in infection control, such as environmental hygiene, crowding, staffing levels and education are inadequate.

\section{Food hygiene}

Milk and meat are also potential sources of various food borne diseases. In 1864 French scientist Louis Pasteur first demonstrated pasteurisation and explained why heat treatment could prevent souring of beer and wine. This process was adapted to preserve other foods, particularly milk ${ }^{[12]}$.

Pasteurization of milk is the process of rapidly heating, maintaining it uniformly over a definite period and rapidly cooling it thus destroying most of the pathogenic microorganisms. Various methods of pasteurizing milk are: Holder (Vat) method, Continuous Flow Method, High Temperature Short Time (HTST) Method, Ultra High Temperature (UHT) Method. Various methods and tests are used to monitor pasteurization.

Prevention of food borne infections and food poisoning can be achieved through appropriate steps taken at the all levels of food processing i.e., production, supply, procurement, processing of raw food, transport, cooking, storage, distribution and finally serving and eating of food. 


\section{Medical hygiene ${ }^{[13]}$}

Medical hygiene pertains to the hygiene practices related to the administration of medicine and medical care that prevents or minimizes the spread of disease.

\section{Medical hygiene practices include}

- Isolation or quarantine of infectious persons or materials to prevent spread of infection.

- Sterilization of instruments used in surgical procedures.

- Use of protective clothing and barriers, such as masks, gowns, caps, eyewear and gloves.

- Proper bandaging and dressing of injuries.

- Safe disposal of medical waste.

- Disinfection of reusables (i.e., linen, pads, uniforms).

- Scrubbing up, handwashing, especially in an operating room, but in more general health-care settings as well, where diseases can be transmitted.

- Ethanol-based sanitizers.

Most of these practices were developed in the 19th century and were well established by the mid-20th century. Some procedures (such as disposal of medical waste) were refined in response to late-20th century disease outbreaks, notably AIDS and Ebola.

Hahnemann as a Pioneer in the field of hygiene in respect of Organon of Medicine ${ }^{[14]}$.

- \$3: Obstacle to recovery and how to remove them from persons in health.

- \$4: Preserver of health

- \$5: In the investigation of fundamental cause e.g., patient's occupation, mode of living, diet and habit.

- \$7: Disease, from which no manifest exciting or maintaining cause (causa occasionalis) has to be removed1, we can perceive nothing but the morbid symptoms

- \$73: In case of individual type of acute disease. In case of sporadic type of acute disease. Caused by meteoric (climatic) and telluric (physical substances e.g., water, soil) influences \& injurious agents.

- \$77: Inappropriately named chronic diseases- Diseases due to continuous exposure to avoidable noxious influences.

- \$78: A true chronic disease arises from chronic miasm. Most robust constitution, best regulated mode of living, most vigorous energy of the vital force cannot eradicate itself.

- \$81(Footnote 1): Causes of transformation of Psora into Chronic Diseases

- Climate \& physical character of the place

- Neglected/ Delayed/ Excess/ Abuse of physical \& mental training of youth (e.g., Diet, Regimen, Passion, Manner, Habits, Customs etc)

- \$150: A slight alteration in the diet \& regimen will usually suffice to dispel such an indisposition.

- \$204: If we deduct all chronic affections, ailments \& diseases that depend on a persistent unhealthy mode of living.

- \$226: Those mental diseases arising from emotional disturbances should be treated with psychical remedies with appropriate diet \& regimen which will rapidly change into health.

- \$260: Chronic diseases are aggravated by noxious influences \& other diseases causing errors in diet \& regimen.
- \$261: Appropriate regimen in treatment of chronic disease. Active exercise in open air, Nutritious unmedicated food \& drinks.

- \$262: Diet \& Regimen in acute disease: In acute disease desired food $\&$ drinks give the patient palliative relief.

\section{Concept of Development of disease}

Hygienic exciting causes like Food poisoning, pollution and lack of personal and social hygiene come in this group cause acute disease and if continues for prolong period cause development of inappropriately named chronic disease as mention in (\$77-Inappropriately named chronic diseasesDiseases due to continuous exposure to avoidable noxious influences). Such types of Diseases easily controlled by cleanliness. Clean fresh water and soap will abolish dirt, and will reduce fleas and lice which love to dwell in dirt. For the treatment of such type of disease master Hahnemann said in same aphorism "These states of ill health, which persons bring upon themselves, disappear spontaneously, provided no chronic miasm lurks in the body, under an improved mode of living, and they cannot be called true chronic disease"

\section{Friends of Health as Pioneer of Preventive Medicine ${ }^{[15]}$.}

- The writings deal with instruction and advises on matter of public health and personal hygiene.

- He criticizes the prejudices and conceptions of his time.

- He stresses upon the importance of cleanliness and antiinfection measures in towns, doctors and nurses, advices to avoid things which pollute the air, dampness, excessive fumigation.

- He gives special attention to the problems of town life, upbringing of children, necessity of exercise and fresh air.

- This was the first book written in a simple style on common subjects of hygiene, which could be read and utilized by the patients as well as physicians.

\section{The Hygiene hypothesis ${ }^{[16]}$.}

The hygiene hypothesis was first introduced in the late 1980s by David P. Strachan, a professor of epidemiology, in the British Medical Journal. Postulates that an environment with a high incidence of infectious diseases protects against allergic and autoimmune diseases, whereas hygienic surroundings increase the incidence of these disorders. This review examines the evidence in support of the hygiene hypothesis and offers a number of mechanisms that could explain the relation between sanitary conditions and susceptibility to allergic and autoimmune diseases.

The hygiene hypothesis [17] remains a credible but nonspecific explanation for observed variations over time, place and persons at risk for developing atopic allergic disorders. More prospective studies are needed to unravel which infectious agents exert a protective effect and the time period of importance for sensitization. The clinical implications of these advances in our understanding of the etiology of atopic allergic disorders are currently limited. This hypothesis has been expanded today to include commensal and symbiotic intestinal microbes, which are profoundly involved in human immune development, and parasitic helminths, which are also strong therapeutic candidates to protect against immune dysregulation.

In this commentary ${ }^{[18]}$ also arguments that the hygiene hypothesis may apply to COVID-19 susceptibility and also 
that residence in low hygienic conditions acts to train innate immune defences to minimize the severity of infection.

Although relationship of the hygiene hypothesis to hygiene practice has not been proved, it lends strong support to initiatives which seek to improve hygiene practice.

\section{Conclusion}

Today, we know much about the behaviour of thousands of disease-causing organisms and about human cellular defences. But we still do not understand human disease avoidance behaviour.

It has been since this time that we have come to understand the enormous benefit of hygiene in preventing the spread of disease-causing organisms ('pathogens').

It is clear 50 years before Chadwich, Hahnemann published his essay on medical hygiene and sanitarian. Physician should aware about all these things in order to preserve health of human being. Then he will be fulfilling, the criteria of aphorism 4 of Organon.

It must be said to the credit of Bacteriology that it paved the way to rapid acceptance of public health measures and hygiene on a grand scale and the eradication of many of the infectious diseases. The decline in the incidence of infectious diseases, however, was countered by the rise in the incidence of functional disorders abiotrophic changes (degenerations) in the tissues and new growths.

Now-a-days homoeopathy is more applied to restore and maintain individual health; but given a chance and proper facilities this system will also hold its own in the field of public health (much to the greater benefit of the mass in general) A thorough study of his works will detail his views which are pioneer work in this field.

\section{References}

1. Hygiene [Internet]. WHO | Regional Office for Africa. 2021. Available from: https://www.afro.who.int/healthtopics/hygiene

2. Blawar R. Text book of public health and community medicine, Department of community medicine, AFMC, with collaboration of WHO, $1^{\text {st }}$ edition 2009.

3. Curtis V. A natural history of hygiene. A natural history of hygiene. Can J Infect Dis Med Microbiol 2007; 18:11-4.

4. Trueman CN. History of Hygiene Timeline historylearningsite.co.uk. The History Learning Site, 1 Sep 2015. 2 Jun 2021.

5. Hygiene throughout history - Hygiene for Health [Internet]. Hygieneforhealth.org.au. 2021. Available from: https://hygieneforhealth.org.au/history/

6. Green VW. Cleanliness and the health revolution. New York: Soap and Detergent Association 1984. Available from:

URL:http:// ww.sdahq.org/about/order_formjs.html

7. Larson E. Hygiene of the Skin: When Is Clean Too Clean? Emerging Infectious Diseases 2001;7(2):225230.

8. Larson E. Social and economic impact of infectious diseases- United States. Clin Performance and the Quality of Health Care 1997;5:31-7.

9. Jumma PA. Hand hygiene: simple and complex doi:10.1016/j.ijid.2004.05.005

10. K. Park's Textbook of Preventive and Social Medicine. $24^{\text {th }}$ ed. Jabalpur: M/s Banarsidas Bhanot 2017.

11. Umaa P. Hand hygiene: simple and complex.
International Journal of Infectious Diseases 2005;9(1):3-14.

12. Hygiene throughout history - Hygiene for Health [Internet]. Hygieneforhealth.org.au. 2021. Available from: https://hygieneforhealth.org.au/history/

13. Hygiene - Wikipedia https://en.wikipedia.org/wiki/Hygiene (page was last edited on 20 May 2021, at 07:26 (UTC)

14. Hahnemann, Samuel, Organon of Medicine. Translated from the fifth edition with an appendix by R.E. Dudgeon, with additions and alterations as per sixth edition translated by William Boericke and introduction by James Krauss; Low Priced Edition; Published by B. Jain Publishers (P) Ltd., New Delhi 2002.

15. Dudgeon RE. The Lesser Writings of Samuel Hahnemann, 14th Impression: New Delhi: B. Jain Publishers Pvt. Ltd 2019.

16. Bach JF. The effect of infections on susceptibility to autoimmune and allergic diseases. N. Engl. J Med 2002;347:911-920.

17. Sheikh, Aziza, Strachan. David $\mathrm{Pb}$ The hygiene theory: fact or fiction?, Current Opinion in Otolaryngology \& Head and Neck Surgery 2004;12(3):232-236. Doi: 10.1097/01.moo.0000122311.13359.30

18. Sehrawat S, Rouse B. Does the hygiene hypothesis apply to COVID-19 susceptibility? Microbes and Infection 2020;22(9):400-402. 